Internat. J. Math. \& Math. Sci.

vol. 3 No. 3 (1980) 559-574

\title{
REPRESENTATION THEORY OF \\ FINITE ABELIAN GROUPS APPLIED TO A LINEAR DIATOMIC CRYSTAL
}

\author{
J.N. BOYD \\ Department of Mathematical Sciences \\ Virginia Commonwealth University \\ Richmond, Virginia 23284 U.S.A. \\ P.N. RAYCHOWDHURY \\ Department of Mathematical Sciences \\ Virginia Commonwealth University \\ Richmond, Virginia 23284 U.S.A.
}

(Received November 21, 1979)

ABSTRACT. After a brief review of matrix representations of finite abelian groups, projection operators are defined and used to compute symmetry coordinates for systems of coupled harmonic oscillators. The Lagrangian for such systems is discussed in the event that the displacements along the symmetry coordinates are complex. Lastly, the natural frequencies of a linear, diatomic crystal are determined through application of the Born cyclic condition and the determination of the symmetry coordinates.

KEY WORDS AND PHRASES. Abelian groups, Born cyclic condition, Group representations, Harmonic oscillators, Lagrangian mechanics, Natural Frequencies, Projection operators. 1980 MATHEMATICS SUBJECT CLASSIFICATION CODES: $20035,70 \mathrm{J10}$. 


\section{INTRODUCTION.}

A classic application of abstract algebra is found in the use of the representation theory of finite groups in the separation of the equations of motion for symmetrically coupled harmonic oscillators. Although the literature on the subject is extensive [3], [4], [5], [6], [9], we shall present a brief review of certain pertinent facts. We shall define projection operators in the event that the group under consideration is abelian and use the projection operators to compute the symmetry coordinates for a physical system. Thereafter we shall discuss the effect of the transformation to symmetry coordinates upon a Lagrangian function. Finally, we shall find the natural frequencies of a one dimensional, diatomic crystal.

\section{GROUP REPRESENTATIONS AND PROJECTION OPERATORS.}

Let $S$ denote a system of finitely many coupled, harmonic oscillators, and let $G$ be a finite symmetry group acting on $S$. Then, if $M(n)$ denotes the multiplicative group of $\mathrm{n} \times \mathrm{n}$ matrices over the complex numbers, $\mathbb{C}$, and if $\Gamma: G \rightarrow M(n)$ is a homomorphism of groups, the image of $\Gamma$, given by $\Gamma(G)=\left\{\Gamma(g) \mid g_{\varepsilon} G\right\}$, is a matrix representation of dimension $n$ for the symmetry group, G.

If $\Gamma(G)$ is unitary and if there exists no similarity transformation to convert $\Gamma(G)$ into the block diagonal form $\underset{j=1}{\operatorname{m}} \Gamma_{j}(G)$ where $\Gamma_{j}(G)$ is a matrix representation of $G$ with dimension less than $n$ for each $j$, then $\Gamma(G)$ is said to be a unitary, irreducible matrix representation of $G$. The number of nonequivalent, unitary, irreducible matrix representations is finite. In fact, there are precisely as many of these as there are conjugacy classes in $G$. If there are $\ell$ conjugacy classes in $G$ and if the dimensions of the nonequivalent, unitary, irreducible matrix representations are $n(1), n(2), \ldots, n(l)$, we know that 


$$
\sum_{j=1}^{\ell}(n(j))^{2}=|G|
$$

where $|G|$ is the order of the finite group [6].

If $G$ is abelian, then $\ell=|G|$ and $n(j)=1$, implying that a11 irreducible representations will be one dimensional. This fact is quite useful. Indeed, if the full symmetry group of the system, $S$, is nonabelian, it is often desirable to choose an abelian subgroup of the larger group to act as $G$, thereby obtaining significant advantages in the computational procedures.

EXAMPLE 2.1. Let $G=\left\{E, R, R^{2}, R^{3}\right\}$ be the cyclic four-group generated by $\mathrm{R}$, a $90^{\circ}$ rotation about a fixed axis. There are four irreducible, unitary representations:

$$
\begin{aligned}
& \Gamma_{1}(G): \Gamma_{1}(E)=\Gamma_{1}(R)=\Gamma_{1}\left(R^{2}\right)=\Gamma_{1}\left(R^{3}\right)=1 ; \\
& \Gamma_{2}(G): \Gamma_{2}(E)=\Gamma_{2}\left(R^{2}\right)=1, \Gamma_{2}(R)=\Gamma_{2}\left(R^{3}\right)=-1 ; \\
& \Gamma_{3}(G): \Gamma_{3}(E)=1, \Gamma_{3}(R)=i, \Gamma_{3}\left(R^{2}\right)=-1, \\
& \Gamma_{3}\left(R^{3}\right)=-i ; \\
& \Gamma_{4}(G): \Gamma_{4}(E)=1, \Gamma_{4}(R)=-i, \Gamma_{4}\left(R^{2}\right)=-1, \\
& \Gamma_{4}\left(R^{3}\right)=i .
\end{aligned}
$$

Suppose that there is associated with each oscillator in $S$ a point mass, $\mathrm{m}_{i}$. If the displacement of the $i-t h$ mass is taken to be $\vec{v}_{i}$, the sma1lest vector space containing all linear combinations of these displacement vectors for all particles is the solution space, $\mathcal{W}$, of the physical system, $S$.

Now for each element, $g$, of the symmetry group, $G$, there is induced exactly one linear operator, $\phi(g)$, which acts on the basis vectors of $q$. 
Furthermore, it can then be shown that $A(\phi(G))=\left\{\sum_{g \in G} a_{g} \phi(g) \mid a_{g} \varepsilon \mathbb{d}\right\}$ is an algebra over $\mathbb{a}$.

In $A(\phi(G))$, there is a subset of operators upon which we fix attention.

DEFINITION 2.1. Let $G$ be abelian and let $\Gamma_{i}(g)$ belong to $\Gamma_{i}(G)$, the i-th unitary, irreducible, one dimensional representation of $G$. Then the linear operator $P_{i} \varepsilon A(\phi(G))$ is defined by $P_{i}=\sum_{g \in G}\left(\Gamma_{i}(g)\right) \phi(g)$, and $\left\{P_{i}|i=1,2, \ldots| G \mid,\right\}$ is the set of projection operators in the algebra $A(\phi(G))$. We emphasize that, in this definition, $G$ is abelian. The reader may easily find the general definition of the projection operators when $G$ is not required to be abelian [1], [9], [10].

EXAMPLE 2.2. $G=\left\{E, R, R^{2}, R^{3}\right\}$ as in Example 2.1. Then the projection operators are

$$
\begin{aligned}
& P_{1}=\phi(E)+\phi(R)+\phi\left(R^{2}\right)+\phi\left(R^{3}\right), \\
& P_{2}=\phi(E)-\phi(R)+\phi\left(R^{2}\right)-\phi\left(R^{3}\right), \\
& P_{3}=\phi(E)+i \phi(R)-\phi\left(R^{2}\right)-i \phi\left(R^{3}\right), \text { and } \\
& P_{4}=\phi(E)-i \phi(R)-\phi\left(R^{2}\right)+i \phi\left(R^{3}\right) .
\end{aligned}
$$

Now suppose that the solution space, $\mathscr{W}$, has the orthonormal basis $B=\left\{\hat{u}_{1}, \hat{u}_{2}, \ldots, \hat{u}_{n}\right\}$. Then the symmetry coordinates for the physical system can be obtained from $B$ as indicated in the next definition.

DEFINITION 2.2. Suppose that $\mathrm{P}_{i}\left(\hat{u}_{\ell}\right)$ is not the zero vector. Then the distinct unit vectors of the form

$$
\frac{P_{i}\left(\hat{u}_{\ell}\right)}{\left|P_{i}\left(\hat{u}_{\ell}\right)\right|}
$$

are the symmetry coordinates of the physical system. 
There are exactly $\mathrm{n}=\mathrm{dim} \mathcal{W}$ distinct nonzero vectors which can be obtained from this definition, and these are mutually orthogonal [1]. This set of vectors establishes a new basis for $\mathcal{O}$ matching the symmetry of $S$ and determines the transformation of coordinates which will separate the equations of motion to the greatest extent possible through the use of symmetry alone. We denote the new basis of symmetry coordinates by $\left\{\hat{e}_{1}, \hat{e}_{2}, \ldots, \hat{e}_{n}\right\}$ and to exhibit their use, we give a simple example.

EXAMPLE 2.3. Four identical masses, $m$, are uniformly spaced and interconnected by identical springs on a frictionless, circular loop fixed in a plane. The spring constant for each spring is $k$. All motion of the masses is confined to the loop. We find the natural frequencies of motion [1], [2].

SOLUTION: The physical system is shown below. The symmetry group is taken to be the rotation group $G=\left\{E, R, R^{2}, R^{3}\right\}$.

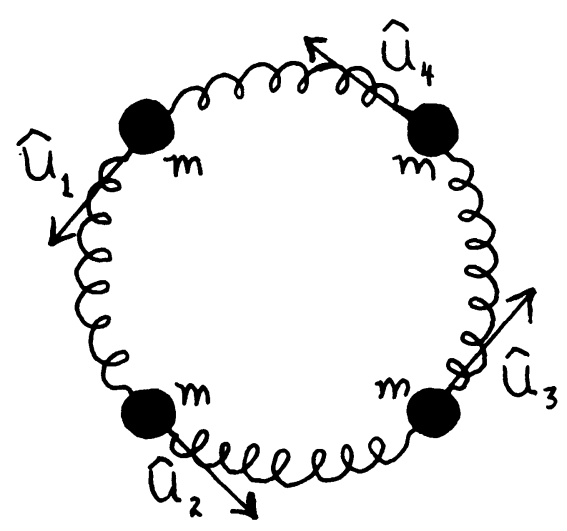

The basis for the solution space is the set of unit tangents $\left\{\hat{u}_{1}, \hat{u}_{2}, \hat{u}_{3}, \hat{u}_{4}\right\}$. The projection operators are given in Example 2.2 and the symmetry coordinates are 


$$
\begin{aligned}
\hat{e}_{1} & =\frac{1}{2} P_{1}\left(\hat{u}_{1}\right)=\frac{1}{2}\left[\phi(E)+\phi(R)+\phi\left(R^{2}\right)+\phi\left(R^{3}\right)\right] \hat{u}_{1} \\
& =\frac{1}{2}\left(\hat{u}_{1}+\hat{u}_{2}+\hat{u}_{3}+\hat{u}_{4}\right), \\
\hat{e}_{2} & =\frac{1}{2}\left(\hat{u}_{1}-\hat{u}_{2}+\hat{u}_{3}-\hat{u}_{4}\right), \\
\hat{e}_{3} & =\frac{1}{2}\left(\hat{u}_{1}+\hat{u}_{2}-\hat{u}_{3}-\hat{i u}_{4}\right), \text { and } \\
\hat{e}_{4} & =\frac{1}{2}\left(\hat{u}_{1}-i \hat{u}_{2}-\hat{u}_{3}+\hat{i}_{4}\right) .
\end{aligned}
$$

These equations serve to define the unitary, transformation matrix $U$ such that

$$
\operatorname{col}\left(\hat{e}_{1} \hat{e}_{2} \hat{e}_{3} \hat{e}_{4}\right)=U \operatorname{col}\left(\hat{u}_{1} \hat{u}_{2} \hat{u}_{3} \hat{u}_{4}\right)
$$

Thus

$$
U=\frac{1}{2}\left(\begin{array}{rrrr}
1 & 1 & 1 & 1 \\
1 & -1 & 1 & -1 \\
1 & i & -1 & -i \\
1 & -i & -1 & i
\end{array}\right)
$$

If the displacements of the masses along the unit tangents are $\mathrm{x}_{1}, \mathrm{x}_{2}, \mathrm{x}_{3}, \mathrm{x}_{4}$ and their velocities are $\dot{x}_{1}, \dot{x}_{2}, \dot{x}_{3}, \dot{x}_{4}$, we can write the displacements and velocities as the column vectors $x=\operatorname{col}\left(x_{1} x_{2} \quad x_{3} x_{4}\right)$ and $\dot{x}=\operatorname{col}\left(\dot{x}_{1} \dot{x}_{2} \dot{x}_{3} \dot{x}_{4}\right)$.

Then the Lagrangian of the system is $L=\frac{1}{2} m \tilde{\mathrm{X}} T \dot{\mathrm{X}}-\frac{1}{2} \mathrm{kXVX}$ where $\tilde{\dot{X}}$ and $\tilde{\mathrm{X}}$ are row vectors obtained by taking the transposes of $\dot{x}$ and $x$, 
$\mathrm{T}=\left(\begin{array}{cccc}1 & 0 & 0 & 0 \\ 0 & 1 & 0 & 0 \\ 0 & 0 & 1 & 0 \\ 0 & 0 & 0 & 1\end{array}\right)$, and $\mathrm{V}=\left(\begin{array}{rrrr}2 & -1 & 0 & -1 \\ -1 & 2 & -1 & 0 \\ 0 & -1 & 2 & -1 \\ -1 & 0 & -1 & 2\end{array}\right)$.

We next transform the Lagrangian to obtain a function of the displacements, $n_{1}, n_{2}, \eta_{3}, n_{4}$, and velocities $\dot{\eta}_{1}, \dot{\eta}_{2}, \dot{\eta}_{3}, \dot{\eta}_{4}$, with respect to the symmetry coordinates, $\hat{e}_{1}, \hat{e}_{2}, \hat{e}_{3}, \hat{e}_{4}$. Thus

$$
\begin{aligned}
L= & \frac{1}{2} \tilde{m}^{-1} U_{U T U}^{-1} U \dot{X} \\
& -\frac{1}{2} k \tilde{X} U^{-1} U V U^{-1} U X \\
= & \frac{1}{2} \tilde{m}^{*} *\left(U T U^{-1}\right) \dot{N}-\frac{1}{2} k \tilde{N} *\left(U V U^{-1}\right) N
\end{aligned}
$$

where $\mathrm{N}=\operatorname{col}\left(n_{1} n_{2} n_{3} n_{4}\right), \dot{\mathrm{N}}=\operatorname{col}\left(\dot{n}_{1} \dot{n}_{2} \dot{n}_{3} \dot{n}_{4}\right)$ and $\tilde{\mathrm{N}}^{*}, \tilde{\dot{\mathrm{N}}}^{*}$ are the transposed, complex conjugates of $N, \dot{N}$ respectively. Since

$$
\mathrm{UTU}^{-1}=\mathrm{T} \text { and } \mathrm{UVU}^{-1}=\left(\begin{array}{cccc}
0 & 0 & 0 & 0 \\
0 & 4 & 0 & 0 \\
0 & 0 & 2 & 0 \\
0 & 0 & 0 & 2
\end{array}\right) \text {, }
$$

we have

$$
\begin{aligned}
& L=\frac{1}{2} \mathrm{~m}\left(\ddot{n}_{1}^{*} \dot{n}_{1}+\ddot{n}_{2}^{*} \dot{n}_{2}+\ddot{n}_{3}^{*} \dot{n}_{3}+\ddot{n}_{4}^{*} \dot{n}_{4}\right) \\
& -\frac{1}{2} k\left(4 n_{2}^{*} n_{2}+2 n_{3}^{*} n_{3}+2 n_{4}^{*} n_{4}\right) \text {. }
\end{aligned}
$$

The completely separated equations of motion are given by

$$
\frac{d}{d t}\left(\frac{\partial L}{\partial \dot{n}_{j}}\right)-\frac{\partial L}{\partial n_{j}}=0
$$


or equivalently by

$$
\frac{d}{d t}\left(\frac{\partial L}{\partial \eta^{*} \underset{j}{*}}\right)-\frac{\partial L}{\partial n_{j}^{*}}=0
$$

for $j \varepsilon\{1,2,3,4\}$.

It follows immediately that the four natural frequencies for this system are

$$
0, \frac{1}{2 \pi} \sqrt{\frac{2 k}{m}}, \frac{1}{2 \pi} \sqrt{\frac{2 k}{m}}, \frac{1}{\pi} \sqrt{\frac{k}{m}} .
$$

\section{THE LAGRANGIAN WITH COMPLEX COORDINATES.}

In the preceding example, the transformation to symmetry coordinates yielded

a real-valued Lagrangian function in the complex variables $\eta_{j}, n_{j}^{*}$. The similarity transformations of the $T$ and $V$ matrices leave their eigenvalues undisturbed. As these eigenvalues, in fact, determine the natural frequencies, such transformations are quite permissible. However, we also wish to show that in the equations of motion as given by Equations 2.1 and 2.2, the variables $n_{j}$ and their complex conjugates, $n_{j}^{*}$, will never appear together. To that end, let us consider the following exercise in partial differentiation.

Let $F$ be a function of the independent complex variables $n_{j}=x_{j}+i y_{j}$. Furthermore, suppose that $F$ is differentiable with respect to $x_{j}$ and $y_{j}$. If $\frac{\partial x_{j}}{\partial y_{i}}=\frac{\partial y_{i}}{\partial x_{j}}=0$ and if we apply the chain rule for partial derivatives while noting that $n_{j}^{*}=x_{j}-i y_{j}$, we obtain $\frac{\partial F}{\partial x_{j}}=\frac{\partial F}{\partial n_{j}}+\frac{\partial F}{\partial n_{j}^{*}}$ and $\frac{\partial F}{\partial y_{j}}=i \frac{\partial F}{\partial n_{j}}-i \frac{\partial F}{\partial n_{j}^{*}}$.

Formally, we write

$$
\frac{\partial}{\partial x_{j}}=\frac{\partial}{\partial \eta_{j}}+\frac{\partial}{\partial \eta_{j}^{*}} \text { and } \frac{\partial}{\partial y_{j}}=i \frac{\partial}{\partial \eta_{j}}-i \frac{\partial}{\partial \eta_{j}^{*}}
$$

Inverting these operational equations, we have 


$$
\begin{aligned}
& \frac{\partial}{\partial \eta_{j}}=\frac{1}{2}\left(\frac{\partial}{\partial x_{j}}-i \frac{\partial}{\partial y_{j}}\right) \text { and } \\
& \frac{\partial}{\partial \eta_{j}^{*}}=\frac{1}{2}\left(\frac{\partial}{\partial x_{j}}+i \frac{\partial}{\partial y_{j}}\right) \text {. }
\end{aligned}
$$

It immediately follows that $\frac{\partial \eta_{j}}{\partial n_{j}}=\frac{\partial n_{j}^{*}}{\partial n_{j}^{*}}=1$ and $\frac{\partial n_{j}^{*}}{\partial n_{j}}=\frac{\partial \eta_{j}}{\partial n_{j}^{*}}=0$.

Thus, whenever the Lagrangian of a physical system of harmonic oscillators is written in the matrix notation $\mathrm{L}=\frac{1}{2} \mathrm{~m} \tilde{\mathrm{N}} * \mathrm{~T}{ }_{0} \mathrm{~N}-\frac{1}{2} \mathrm{k} \tilde{\mathrm{N}}^{*} \mathrm{~V}_{0} \mathrm{~N}$, each summand of the expanded Lagrangian will be of the form $c_{1} \dot{n} \dot{j}^{*} \dot{\eta}_{k}$ or $c_{2}{ }^{*}{ }_{j}^{*} n_{k}$ where $c_{1}$ and $c_{2}$ are constant.

Thus the differential equation

$$
\frac{d}{d t}\left(\frac{\partial L}{\partial \dot{\eta}_{j}}\right)-\frac{\partial L}{\partial n_{j}}=0 \text { contains }
$$

only the complex conjugates $\eta_{k}^{*}$ together with their second derivatives with respect to time and

$$
\frac{d}{d t}\left(\frac{\partial L}{\partial \dot{n}_{j}^{*}}\right)-\frac{\partial L}{\partial \eta_{j}^{*}}=0 \text { contains }
$$

only the coordinates $\eta_{k}$ and $\ddot{\eta}_{k}$. That is, in neither equation will coordinates and their complex conjugates be mixed. The proper frequencies can be obtained from equations of either form.

\section{THE ONE-DIMENSIONAL DIATOMIC CRYSTAL.}

We now consider a circular loop of alternating masses of two sorts, $m$ and M, connected by identical springs having force constant $B$ [2]. Let there be 2N masses in all with $\mathrm{N}$ of each kind. If $\mathrm{N}$ is taken to be very large, we have essentially applied the Born cyclic condition to a linear, diatomic crystal 
[1], [8] . We will now find the symmetry coordinates and natural frequencies of the system.

The system is invariant with respect to rotations by $\frac{2 \pi}{\mathrm{N}}$, and, in the neighborhood of the $2 n-t h$ and $(2 n+1)-t h$ particles, the circular arrangement appears as shown.

In the calculations to follow, two facts concerning the complex roots of unity are required.
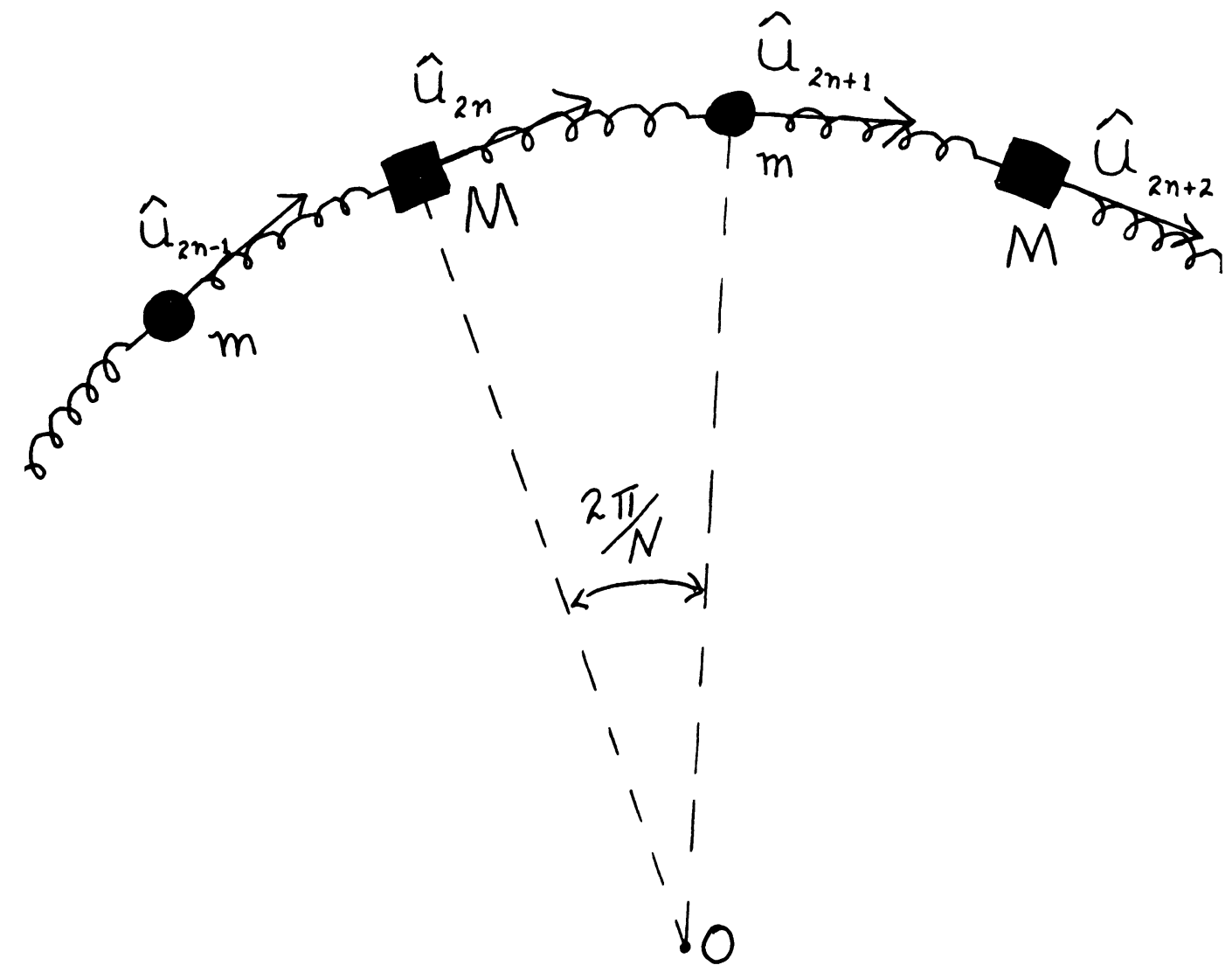

First, let $\exp \frac{2 \pi i}{\mathrm{~K}}$ be the primitive complex $\mathrm{K}$-th root of unity. Then let $\mathrm{n}_{1}$ and $\mathrm{n}_{2} \varepsilon\{0,1,2, \ldots, \mathrm{K}-1\}$. By application of the Parallelogram Law of Addition for Complex Numbers 
$\exp n_{1}\left(\frac{2 \pi i}{K}\right)+\exp n_{2}\left(\frac{2 \pi i}{K}\right)=2\left|\cos \left(\frac{n_{1}-n_{2}}{2}\right) \frac{2 \pi}{K}\right| \exp \left(\frac{n_{1}+n_{2}}{2}\right) \frac{2 \pi i}{K}$. (Equation 4.1) In the event that we need to consider $\exp 2 \pi i+\exp n \frac{2 \pi}{K} i$ where $0 \leq n \leq K-1$, we write

$$
\exp 0 i+\exp n \frac{2 \pi i}{K}=2\left|\cos \frac{n}{2}\left(\frac{2 \pi}{K}\right)\right| \exp \frac{n}{2}\left(\frac{2 \pi i}{K}\right)
$$

Secondly we recall that the sum of the $\mathrm{K}$ complex $\mathrm{K}$-th roots of unity is the complex number zero. That is

$$
\sum_{n=0}^{K-1} \exp n \frac{2 \pi i}{K}=0+0 i, K>1
$$

The symmetry group of the circular arrangement is taken to be $C_{N}$, the group of rotations $\left\{\mathrm{R}\left(\frac{2 \pi \ell}{\mathrm{N}}\right) \mid \ell=1,2,3, \ldots, \mathrm{N}\right\}$. The $\mathrm{N}$ one dimensional representations of the symmetry group are given by

$$
\Gamma_{k}(G): \Gamma_{k}\left(R\left(\frac{2 \pi \ell}{N}\right)\right)=\exp \left(\frac{2 k \pi}{N}\right) \ell i
$$

for $\ell=1,2,3, \ldots, N \quad[1]$.

Thus the projection operators are of the form

$$
P_{k}=\sum_{\ell=1}^{N}\left(\exp \left(\frac{2 k \pi}{N}\right) \ell i\right) \phi\left(R\left(\frac{2 \pi \ell}{N}\right)\right) \text { for } k=1,2,3, \ldots, N
$$

The symmetry coordinates are found by letting $\mathrm{P}_{k}$ act first upon the unit tangent $\hat{\mathrm{u}}_{2 \mathrm{~N}-1}$ and then upon $\hat{\mathrm{u}}_{2 \mathrm{~N}}$.

$$
\hat{\mathrm{e}}_{2 \mathrm{k}-1}=\frac{1}{\sqrt{\mathrm{N}}} \mathrm{P}_{\mathrm{k}} \hat{\mathrm{u}}_{2 \mathrm{~N}-1}=\frac{1}{\sqrt{\mathrm{N}}} \sum_{\ell=1}^{\mathrm{N}}\left(\exp \left(\frac{2 \mathrm{k} \pi}{\mathrm{N}}\right) \ell \mathrm{i}\right) \hat{\mathrm{u}}_{2 \ell-1}
$$

and 
and

$$
\hat{e}_{2 k}=\frac{1}{\sqrt{N}} P_{k} \hat{u}_{2 N}=\frac{1}{\sqrt{N}} \sum_{\ell=1}^{N}\left(\exp \left(\frac{2 k \pi}{N}\right) l i\right) \hat{u}_{2 \ell} \text { for } k=1,2,3, \ldots, N
$$

The transformation matrix $U$ is

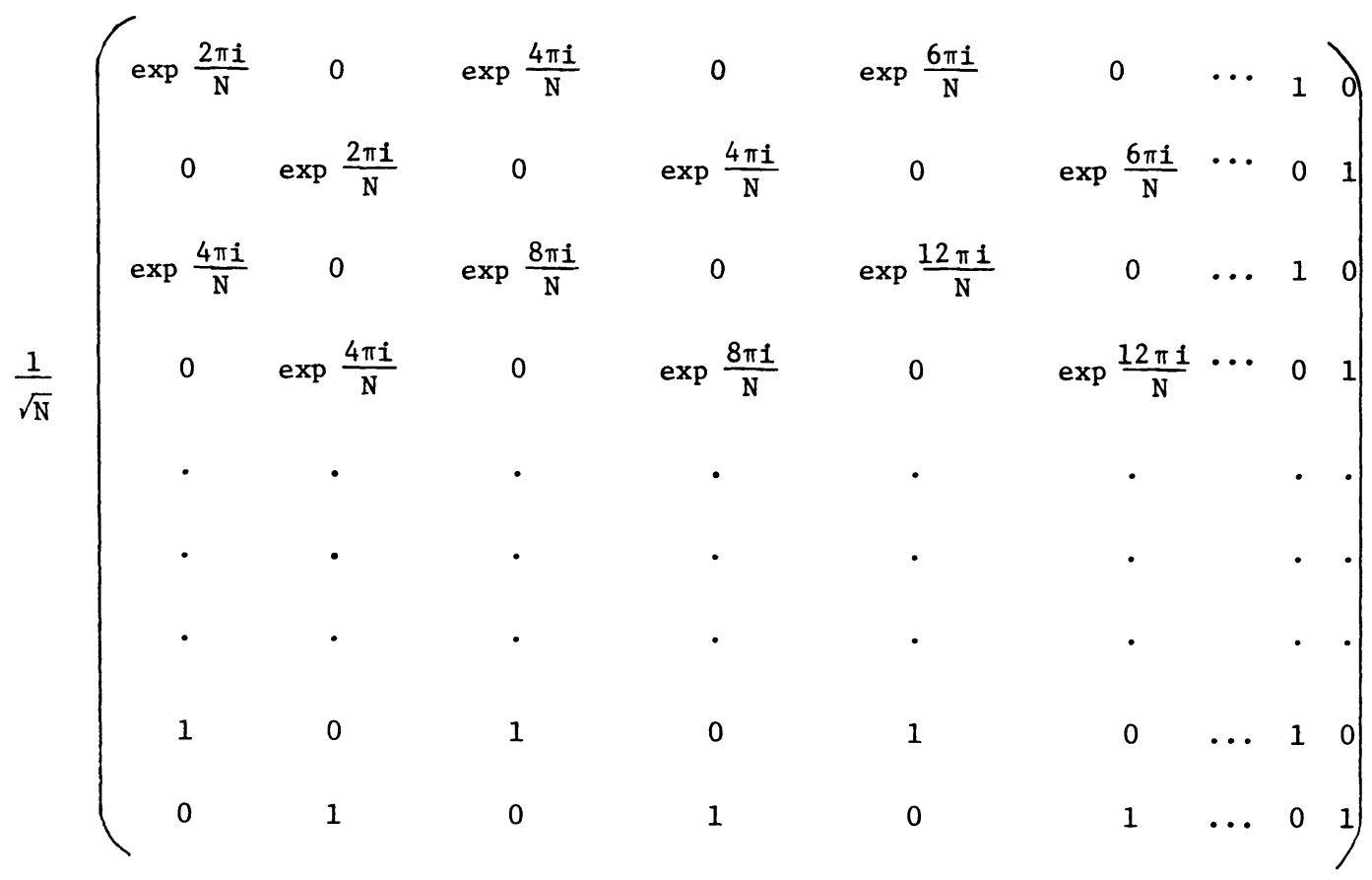

In terms of the displacements and velocities along the unit tangents, the Lagrangian of the system is

$$
\begin{gathered}
L=\frac{1}{2} \dot{\tilde{x} T \dot{X}}-\frac{1}{2} \beta \tilde{X} V x \text { where } x=\operatorname{col}\left(x_{1} x_{2} \ldots x_{2 N}\right), \\
\dot{X}=\operatorname{col}\left(\dot{x}_{1} \dot{x}_{2} \ldots \dot{x}_{2 N}\right)
\end{gathered}
$$




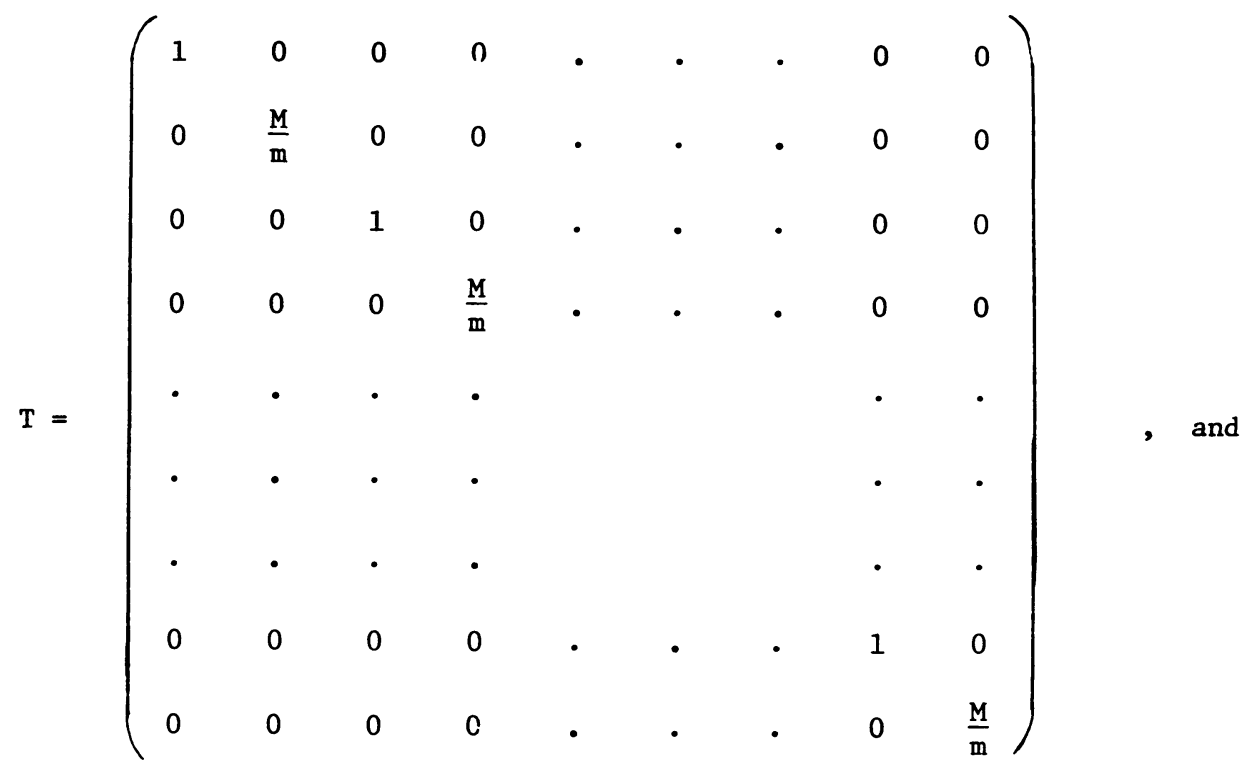

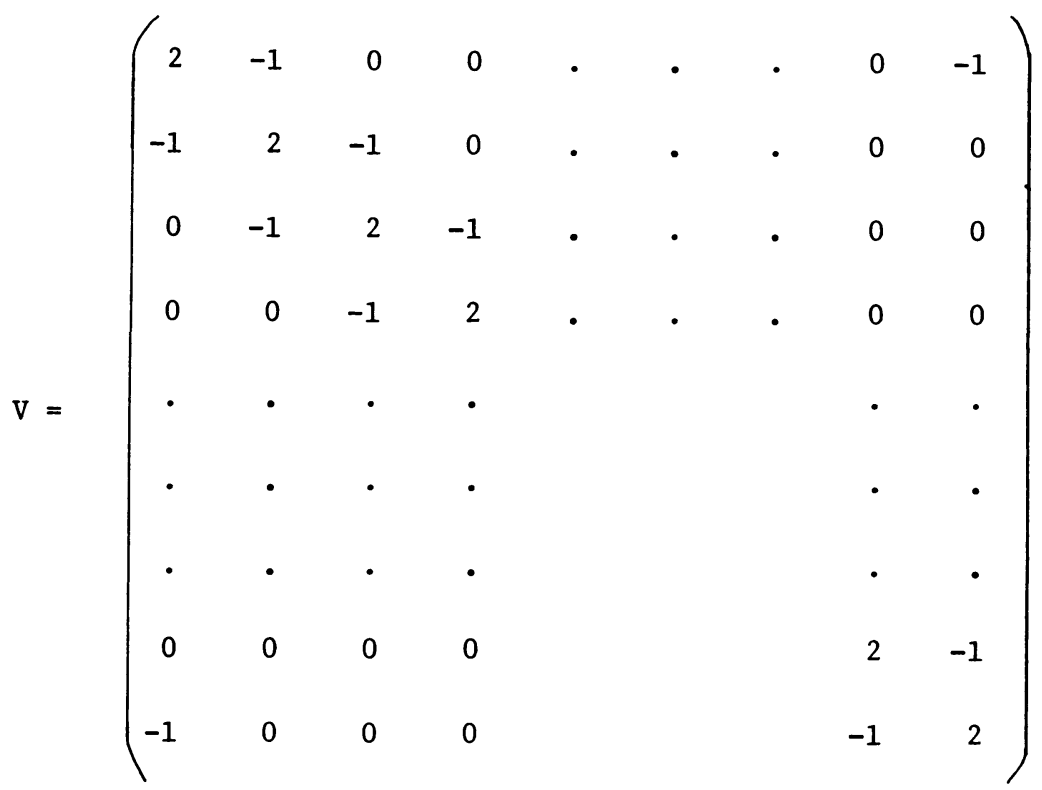


The transformed Lagrangian is

$$
\mathrm{L}=\frac{1}{2} \mathrm{~m} \tilde{\mathrm{N}}^{*}\left(\mathrm{UTU}^{-1}\right) \dot{\mathrm{N}}-\frac{1}{2} \tilde{\mathrm{N}}^{*}\left(\mathrm{UVU}^{-1}\right) \mathrm{N}
$$

where $N=\operatorname{col}\left(n_{1} n_{2} \ldots n_{2 N}\right)$ with respect to the symmetry coordinates. It is easily shown that $\mathrm{UTU}^{-1}=\mathrm{T}$.

The computation of $\mathrm{UVU}^{-1}$ is straightforward but tedious. However, by the use of the identities given in Equations 4.1 and 4.2 , the matrix product becomes

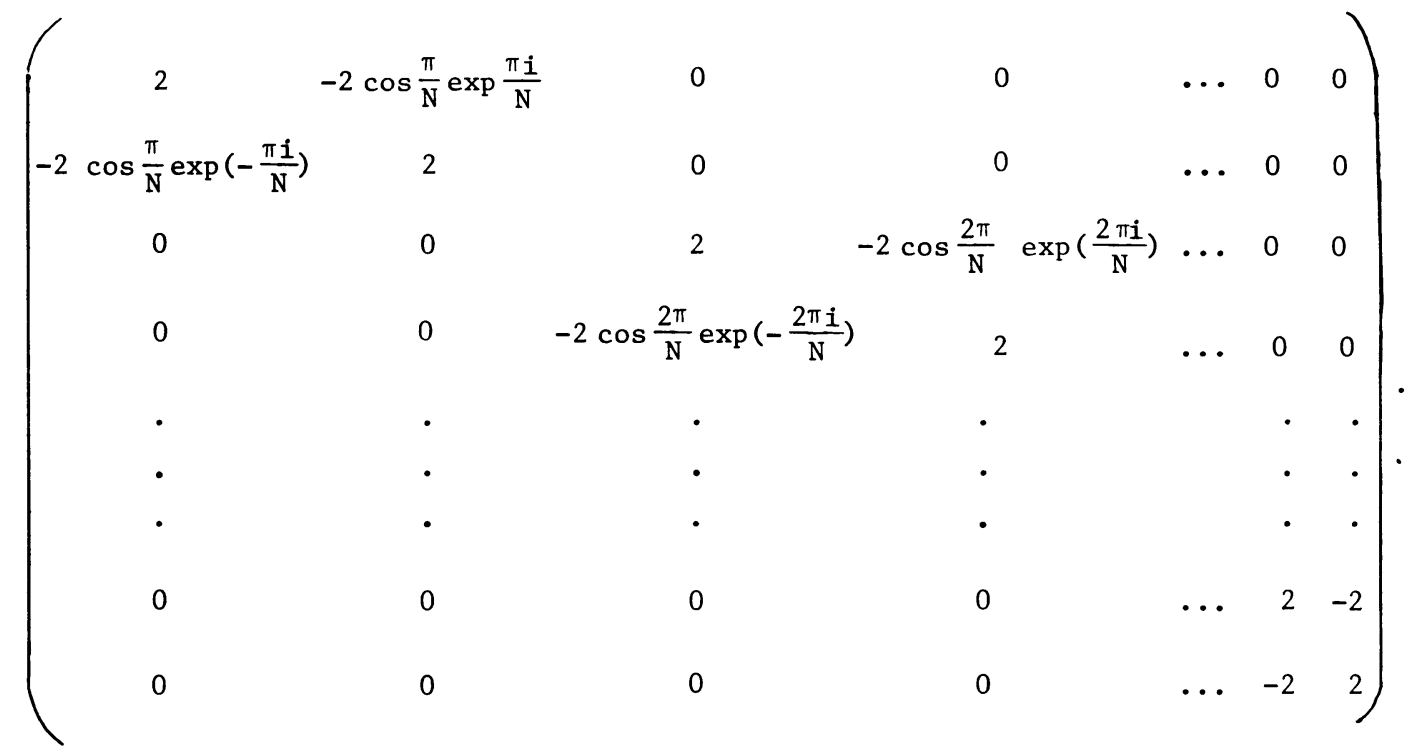

The k-th $2 \times 2$ block along the principal diagonal of the matrix $\mathrm{UVU}^{-1}$ is

$$
\left(\begin{array}{cc}
2 & -2 \cos \frac{k \pi}{N} \exp \left(\frac{k \pi i}{N}\right) \\
-2 \cos \frac{k \pi}{N} \exp \left(-\frac{k \pi i}{N}\right) & 2
\end{array}\right) .
$$

Thus, for $\eta_{2 k-1}$ and $\eta_{2 k}$ we have 


$$
\begin{aligned}
& L\left(n_{2 k-1}, n_{2 k}\right)=\frac{1}{2} m\left(\dot{n}_{2 k-1}^{*} \dot{n}_{2 k}^{*}\right)\left(\begin{array}{ll}
1 & 0 \\
0 & \frac{M}{m}
\end{array}\right)\left(\begin{array}{l}
\dot{n}_{2 k-1} \\
\dot{n}_{2 k}
\end{array}\right) \\
& -\frac{1}{2} \beta\left(n_{2 k-1}^{*} n_{2 k}^{*}\right)\left(\begin{array}{cc}
2 & -2 \cos \frac{k \pi}{N} \exp \left(\frac{k \pi i}{N}\right) \\
-2 \cos \frac{k \pi}{N} \exp \left(-\frac{k \pi i}{N}\right) & 2
\end{array}\right)\left(\begin{array}{l}
n_{2 k}-1 \\
2 k
\end{array}\right) \\
& =\frac{1}{2}\left(m \dot{n}_{2 k-1}^{*} \dot{n}_{2 k-1}+M \dot{n}_{2 k}^{*} \dot{\eta}_{2 k}\right) \\
& -\beta\left(n_{2 k-1}^{*} n_{2 k-1}-\cos \frac{k \pi}{N} \exp \left(-\frac{k \pi i}{N}\right) n_{2 k}^{*} n_{2 k-1}\right. \\
& \left.-\cos \frac{k \pi}{N} \exp \left(\frac{k \pi i}{N}\right) n_{2 k-1}^{*} n_{2 k}+n_{2 k}^{*} n_{2 k}\right) \text {. }
\end{aligned}
$$

The equations of motion are

$$
\begin{aligned}
& \frac{1}{2} m \ddot{\eta}_{2 k-1}+\beta \eta_{2 k-1}-\beta \cos \frac{k \pi}{N} \exp \left(\frac{k \pi i}{N}\right) \eta_{2 k}=0, \\
& \frac{1}{2} M \ddot{n}_{2 k}^{*}+\beta \eta_{2 k}-\beta \cos \frac{k \pi}{M} \exp \left(-\frac{k \pi i}{N}\right) \eta_{2 k-1}=0 .
\end{aligned}
$$

The frequencies satisfy the secular determinant

$$
\begin{aligned}
& \operatorname{det}\left(\begin{array}{l}
-\frac{2 \beta}{m}+4 \pi^{2} f^{2} \\
\frac{2 \beta}{M} \cos \frac{k \pi}{N} \exp \left(-\frac{k \pi i}{N}\right)-\frac{2 \beta}{M}+4 \pi^{2} f^{2} \\
\text { Thus } \quad f=\frac{\sqrt{\beta}}{2 \pi} \sqrt{\left(\frac{1}{m}+\frac{1}{M}\right) \pm \sqrt{\left(\frac{1}{m}+\frac{1}{M}\right)^{2}-\frac{4}{M m} \sin ^{2} \frac{k \pi}{N}}} .
\end{array}=0 .\right.
\end{aligned}
$$

Recognizing that, in solid state physics, the wave number for the vibration is $\mathrm{K}=\frac{\mathrm{k} \pi}{\mathrm{Na}}$, where a is the separation of neighboring particles, the result 
may be rewritten as

$$
f=\frac{\sqrt{\beta}}{2 \pi} \sqrt{\left(\frac{1}{m}+\frac{1}{M}\right) \pm \sqrt{\left(\frac{1}{m}+\frac{1}{M}\right)^{2}-\frac{4 \sin ^{2} \mathrm{Ka}}{M m}}}
$$

The value $K=\frac{\pi}{2 a}$ defines the boundary of the first Brillouin zone in reciprocal lattice space. The familiar forbidden frequency gap appears since, for $K=\frac{\pi}{2 a}$, f will have the separated values

$$
\frac{\sqrt{\beta}}{2 \pi} \sqrt{\frac{2}{m}} \quad \text { or } \quad \frac{\sqrt{\beta}}{2 \pi} \sqrt{\frac{2}{M}} \quad[7]
$$

\section{REFERENCES}

1. Boyd, J.N. and Raychowdhury, P.N., "An Application of Projection Operators to a One Dimensional Crysta1", Bulletin of the Institute of Mathematics, Academia Sinica 7(1979), 133-144.

2. Boyd, J.N. and Raychowdhury, P.N., "Projection Operator Techniques in Lagrangian Mechanics: Symetrically Coupled Oscillators", to appear in International Journal of Mathematics and Mathematical Sciences;

3. Duffey, G.H., Theoretical Physics, Houghton Mifflin Company, Boston, 1973.

4. Gamba, A., "Representations and Classes in Groups of Finite Order", Journal of Mathematical Physics 9 (1968), 186-192.

5. Hamermesh, M., Group Theory and Its Application to Physical Problems, Addison Wesley Publishing Company, Reading, Mass., 1962.

6. Joshi, A.W., Elements of Group Theory for Physicists, Halsted Press, New York, 1973.

7. Kittel, C., Introduction to Solid State Physics (Second Edition), John Wiley and Sons, New York, 1959.

8. Mariot, L., (translated by A. Nussbaum), Group Theory and Solid State Physics Prentice-Ha11, Englewood Cliffs, N.J., 1964.

9. Nussbaum, A., "Group Theory and Normal Modes", American Journal of Physics $36,529-539$.

10. Wilde, R.E., "A General Theory of Symmetry Coordinates", American Journal of Physics 32 (1964), 45-52. 


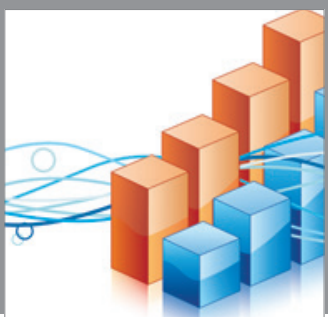

Advances in

Operations Research

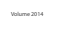

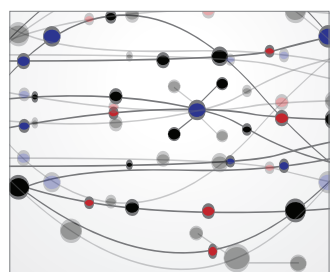

\section{The Scientific} World Journal
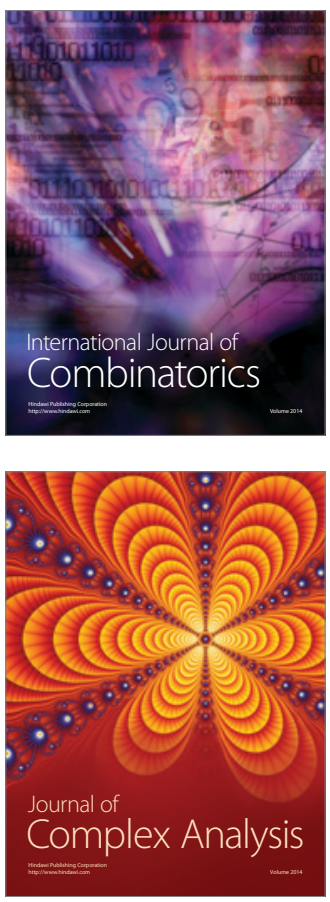

International Journal of

Mathematics and

Mathematical

Sciences
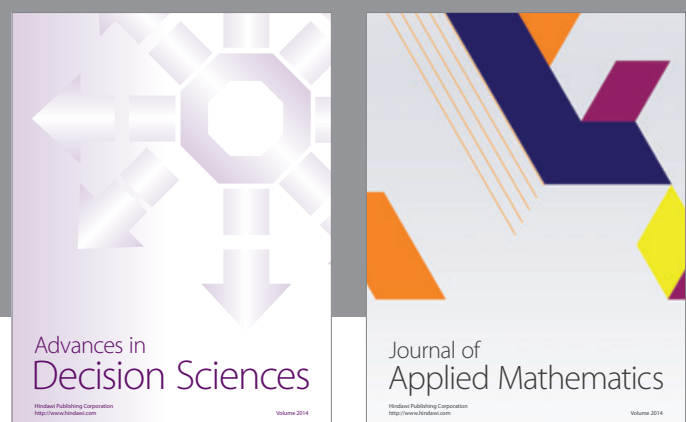

Journal of

Applied Mathematics
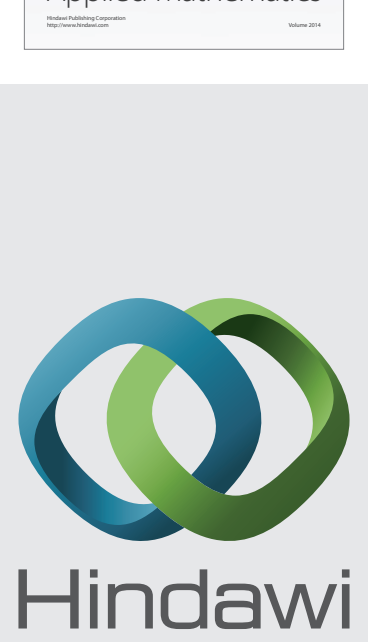

Submit your manuscripts at http://www.hindawi.com
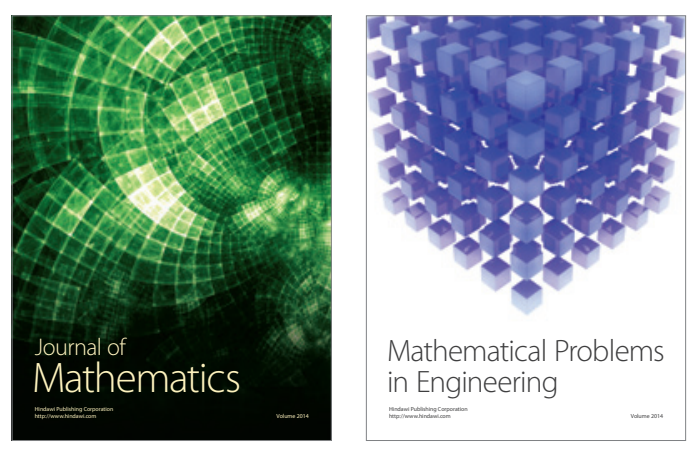

Mathematical Problems in Engineering
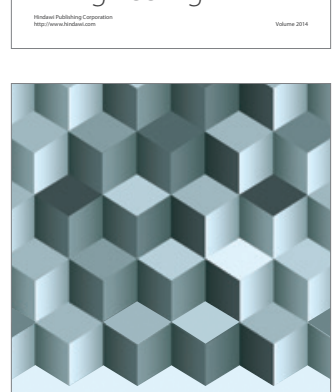

Journal of

Function Spaces
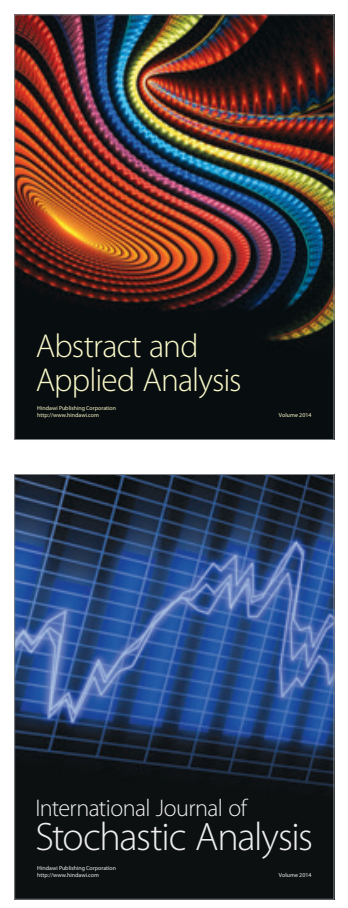

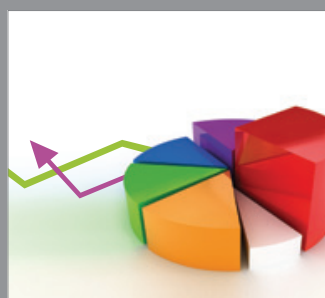

ournal of

Probability and Statistics

Promensencen
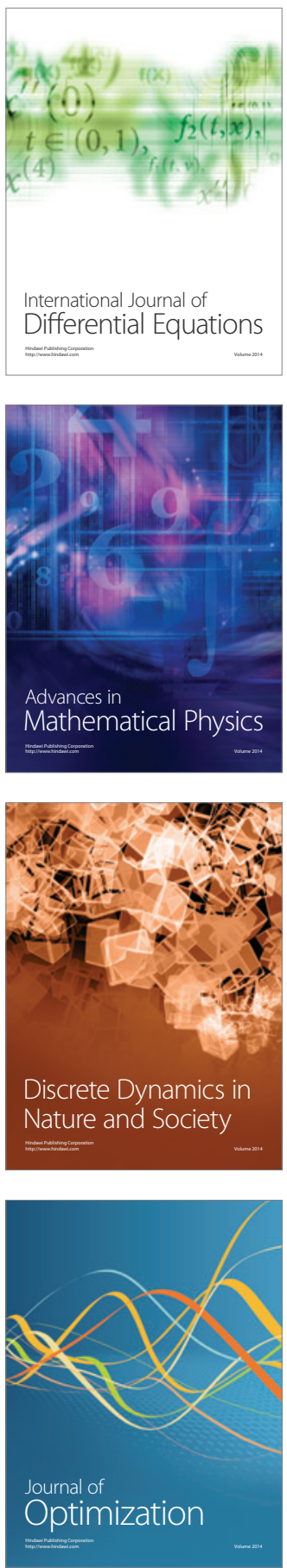